\title{
Erratum: Bright Solitonic Matter-Wave Interferometer [Phys. Rev. Lett. 113, 013002 (2014)]
}

\author{
G. D. McDonald, C. C. N. Kuhn, K. S. Hardman, S. Bennetts, P. J. Everitt, P. A. Altin, \\ J.E. Debs, J. D. Close, and N. P. Robins
}

(Received 23 February 2017; published 26 May 2017)

DOI: 10.1103/PhysRevLett.118.219903

A small error was recently found in the code used in this work to experimentally calibrate the magnetic bias field around the $155 \mathrm{G}$ Feshbach resonance of ${ }^{85} \mathrm{Rb}$. The error occurred when moving from calibrating the magnetic field with ${ }^{85} \mathrm{Rb}$ as used in our previous work to ${ }^{87} \mathrm{Rb}$, due to an error in the Breit-Rabi equation used.

This error in the calibration does not change the experimental results or the conclusions of the Letter, although the axes in the original Figs. 2(a) and 3(b) are labeled incorrectly due to the calibration offset. The corrected calibration puts the soliton parameter at $a_{s}=-2.3$ for the experimental setup used. The optimum scattering length at which the interferometer operates is at the point where a solitonic matter wave is formed, and atomic dispersion is minimized. As can be seen in the updated Fig. 3(b), it is difficult to see this because of the large scattering length scan from $-100 a_{0}$ to $170 a_{0}$. The experiment was therefore repeated using a finer scan of the $s$-wave scattering length around the soliton point. The experimental setup has changed since 2014, including a larger number of atoms in the condensate, a higher repeatability of the experiment, and rebuilt optical traps. This results in a soliton parameter of $a_{s}=-1.2 a_{0}$ in the current experiment. Visibility data are shown in Fig. E1 (originating in this Erratum) for a $T=2 \mathrm{~ms}$ interferometer and $N=3 \times 10^{4}$ Bose-Einstein-condensed ${ }^{85} \mathrm{Rb}$ atoms. While the new data confirm the conclusions of the Letter, the effect is less pronounced.

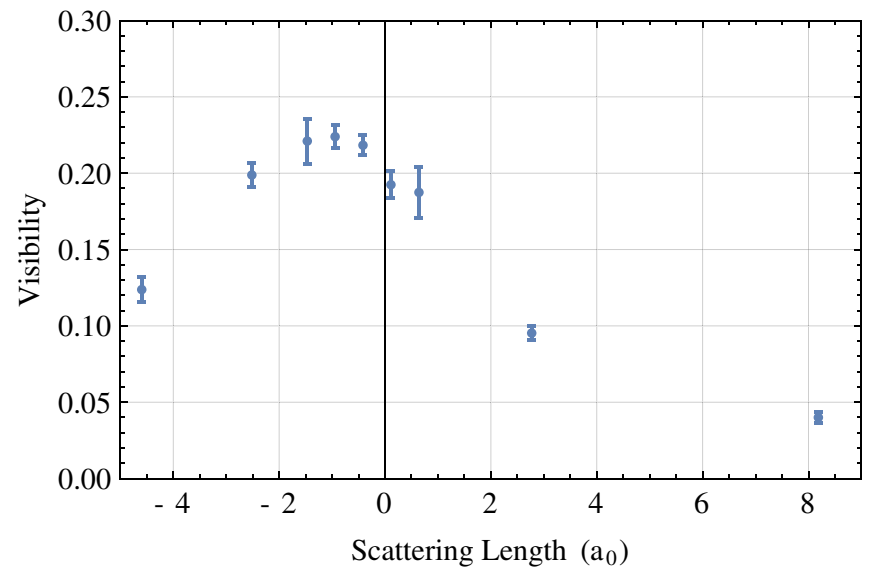

FIG. E1. Fringe visibility as a function of the $s$-wave scattering length $a$ for a $T=2 \mathrm{~ms}$ Mach-Zehnder atom interferometer in an optical waveguide.

Published by the American Physical Society under the terms of the Creative Commons Attribution 3.0 License. Further distribution of this work must maintain attribution to the author(s) and the published articles title, journal citation, and DOI. 


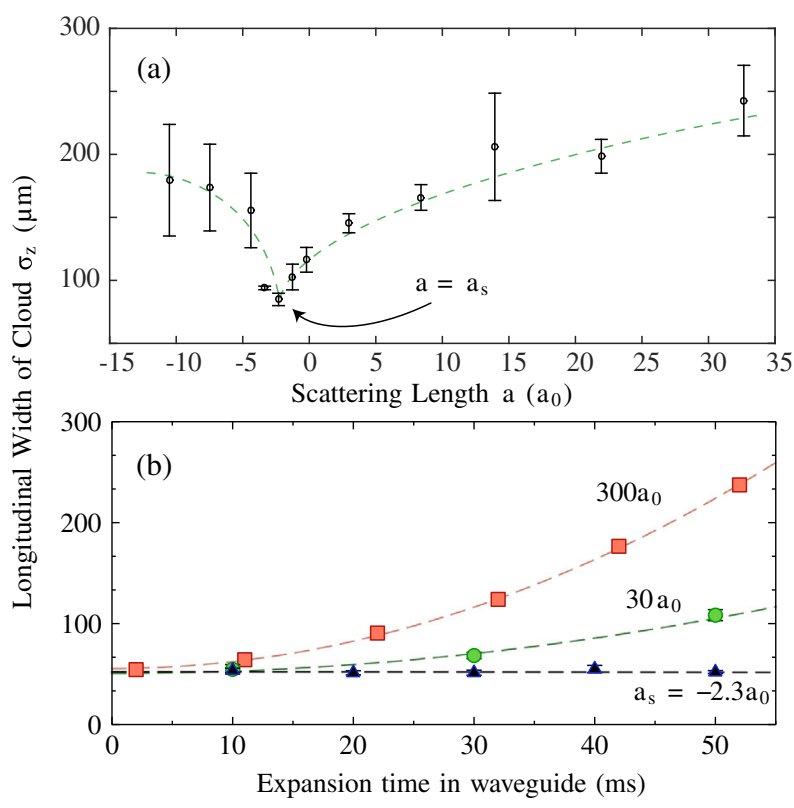

FIG. 2. Figure from original manuscript. (a) Longitudinal width of a matter wave as a function of the scattering length, measured after $90 \mathrm{~ms}$ of free expansion in the guide. The green dashed lines are to guide the eye. The soliton parameter $a_{s}=-2.3 a_{0}$ is seen to minimize this expansion. (b) Comparison of the longitudinal expansion along the guide for three different scattering lengths: the repulsive selfinteraction of $a=300 a_{0}$, the low interaction case of $a=30 a_{0}$, and the soliton parameter $a_{s}=-2.3 a_{0}$. The dashed lines are parabolic fits to extract the acceleration of the cloud width. For $a=a_{s}$ this acceleration is consistent with zero. The error bars shown in both (a) and (b) are statistical.
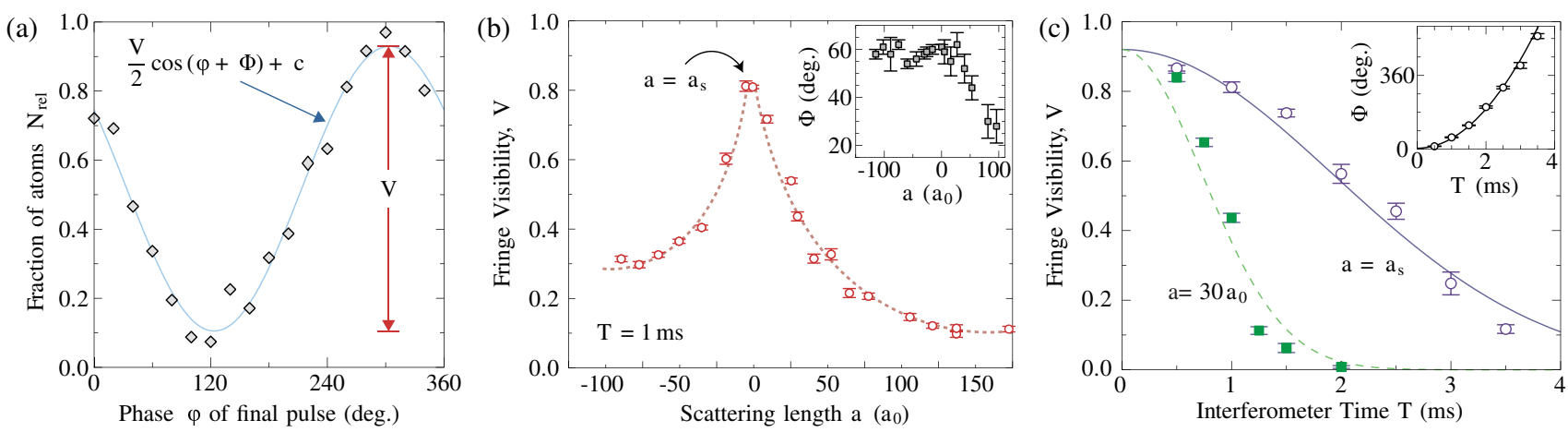

FIG. 3. Figure from original manuscript. (a) Each interferometric fringe is fitted to a function $N_{\text {rel }}=(\mathcal{V} / 2) \cos (\phi+\Phi)+c$ to extract the fringe visibility $\mathcal{V}$ and interferometric phase $\Phi$. The data shown here are the interference fringe for a soliton, with $a=a_{s}$. (b) Fringe visibility (open circles) as a function of the $s$-wave scattering length $a$ for a $T=1 \mathrm{~ms}$ Mach-Zehnder atom interferometer. The red dashed lines are to guide the eye. A dramatic increase in the fringe visibility is seen at the soliton parameter $a_{s} \approx-2.3 a_{0}$ for our system. Inset: the interferometric phase $\Phi$ for various $a$. (c) Fringe visibility as a function of the interferometer time $T$. The results for a weakly interacting interferometer with $a=30 a_{0}$ (filled green squares), and the soliton interferometer with $a=a_{s}=-2.3 a_{0}$ (open circles) are shown. The Gaussian fits provide half-maximum decay times of $0.9 \mathrm{~ms}$ (green dashed line) and $2.3 \mathrm{~ms}$ (solid purple line), respectively. The solitonic matter-wave optimizes the fringe visibility at all times we have tested. Inset: measured interferometric phase $\Phi$ for the soliton interferometer as a function of $T$, along with a quadratic fit. All uncertainties in (b) and (c) are $1 \sigma$ confidence intervals of the fit to each interferometric fringe.

The authors gratefully acknowledge the assistance of Mahasen Sooriyabandara, Simon Cornish, and Catherine Klauss for helping isolate and remove this error. 